\title{
LAS MEJORES PRÁCTICAS \\ DE LAS ORGANIZACIONES \\ Y LOS PROFESIONALES
}

\section{Professional and Organizational Best Practices}

\author{
Robert L. SchalOcK, PHD. \\ Professor Emeritus. Hastings College, P.O. Box 285, Chewelah, WA 99109-0285. USA \\ rschalock@ultraplix.com
}

Recepción: 26 de noviembre de 2014

Fecha de aceptación definitiva: 1 de diciembre de 2014

Biblid. [0210-1696 (2015) vol. 46 (1), n. ${ }^{\circ} 253$, enero-marzo; 7-23]

RESUMEN: Este artículo se centra en las prácticas basadas en la evidencia y, sobre esta base, hacemos una invitación a que busquemos entre todos cuáles son las mejores prácticas, quién es el profesional más adecuado y qué significa ser una organización eficaz y eficiente. Tanto los profesionales como las organizaciones ofrecen servicios y apoyos que contribuyen a mejorar el bienestar y el crecimiento personal de los usuarios. En el contexto de la discusión sobre las prácticas profesionales y de las organizaciones, indicaré que las mejores prácticas profesionales empiezan con el respeto hacia las personas, el cumplimiento de los estándares y las normas éticas y deontológicas, y la utilización de las prácticas basadas en la evidencia y de la evaluación del efecto y de los resultados conseguidos. Indicaré, asimismo, que las mejores prácticas de la organización empiezan con el compromiso de ser una entidad basada en valores y, a la vez, de ser eficaz y eficiente en la prestación de los servicios y apoyos. Este compromiso de la organización se refleja en el uso de las mejores prácticas, que consisten en la utilización de equipos de alto rendimiento, en el paradigma de los apoyos, en la evaluación de los resultados obtenidos y en la mejora continua de la calidad.

Como se observa en la Figura 1, examinaremos cada uno de los componentes de las mejores prácticas profesionales y de las organizaciones. Además, indicaré que, a través de su acción recíproca, las mejores prácticas de los profesionales y de las organizaciones generan un entorno cultural que tiene un efecto directo, no solo en la mejora de los servicios y apoyos que la organización ofrece a los usuarios, sino también en el bienestar y crecimiento personal del personal de la organización, lo cual, a su vez, redunda en un aumento de su eficacia y eficiencia. 
Palabras Clave: prácticas basadas en la evidencia; apoyos; equipos de alto rendimiento.

AвSTRACT: By focusing on evidence-based practices this article asks us to pursue jointly what are best practices, who is a professional, and what does it mean to be an effective and efficient organization. Both professionals and organizations provide services and supports that enhance the personal well-being and personal growth of their clientele. In discussing professional and organizational practices, I will suggest that professional best practices begin with respect for the individual and embrace professional standards, professional ethics, evidence-based practices, and impact evaluation. Analogously, I will suggest that organization best practices begin with a commitment to being a values-based entity that is effective and efficient in the provision of services and supports. This organization commitment is reflected in best practices related to high performance teams, the supports paradigm, outcomes evaluation, and continuous quality improvement.

As depicted in Figure 1, the presentation will discuss each of these components of professional and organizational best practices. Additionally, I will suggest that through their reciprocal action, the best practices exhibited by professionals and organizations also create a cultural milieu that directly enhances not only the services and supports provided to the organization's clientele, but also directly impacts the personal wellbeing and growth of organization personnel, which in turn enhances their effectiveness and efficiency.

KEY WORDS: evidence-based practices; supports; high performance teams.

\section{Introducción y cuestiones generales}

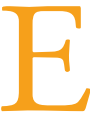

STE ARTíCULO SE CENTRA EN LAS PRÁCTICAS BASADAS EN LA EVIDENCIA y, sobre esta base, hacemos una invitación a que busquemos entre todos cuáles son las mejores prácticas, quién es el profesional más adecuado y qué significa ser una organización eficaz y eficiente. Tanto los profesionales como las organizaciones ofrecen servicios y apoyo que contribuyen a mejorar el bienestar y el crecimiento personal de los usuarios. En el contexto de la discusión sobre las prácticas profesionales y de las organizaciones, indicaré que las mejores prácticas profesionales empiezan con el respeto hacia las personas; el cumplimiento de los estándares y las normas éticas y deontológicas; y la utilización de las prácticas basadas en la evidencia y de la evaluación del efecto y de los resultados conseguidos. Indicaré, asimismo, que las mejores prácticas de la organización empiezan con el compromiso de ser una entidad basada en valores y, a la vez, de ser eficaz y eficiente en la prestación de los servicios y apoyos. Este compromiso de la organización se refleja en el uso de las mejores prácticas, que consisten en la utilización de equipos de alto rendimiento, en el paradigma de los apoyos, en la evaluación de los resultados obtenidos y en la mejora continua de la calidad.

Como se observa en la Figura 1, examinaremos cada uno de los componentes de las mejores prácticas profesionales y de las organizaciones. Además, indicaré que, a

(C) Ediciones Universidad de Salamanca

Siglo Cero, vol. 46 (1), n。 253, 2015, enero-marzo, pp. 7-23 
través de su acción recíproca, las mejores prácticas de los profesionales y de las organizaciones generan un entorno cultural que tiene un efecto directo, no solo en la mejora de los servicios y apoyos que la organización ofrece a los usuarios, sino también en el bienestar y crecimiento personal del personal de la organización, lo cual, a su vez, redunda en un aumento de su eficacia y eficiencia.

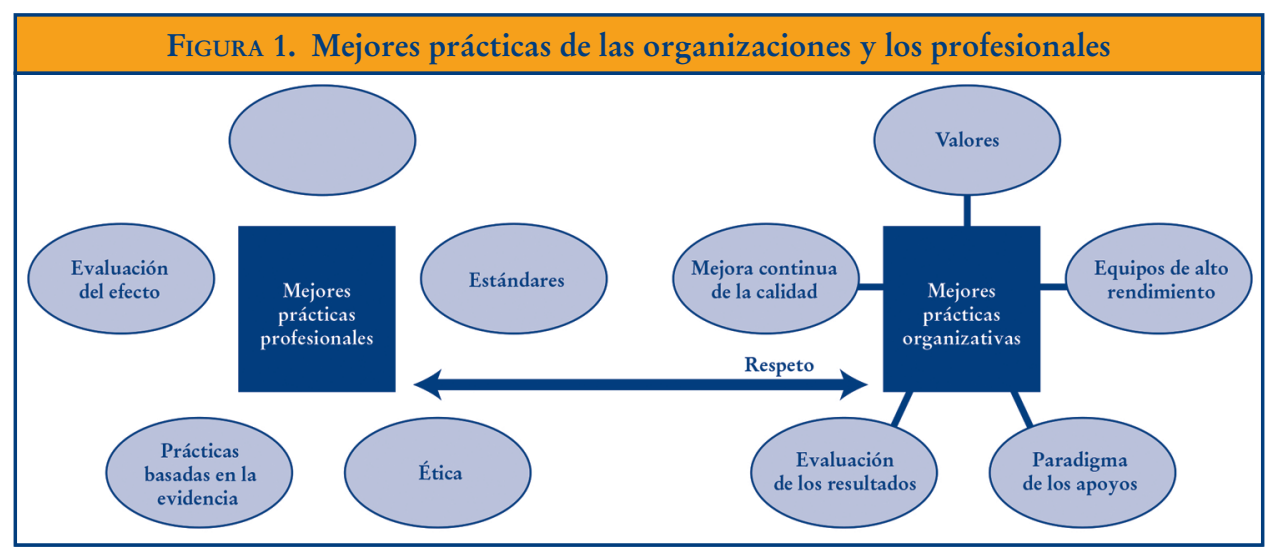

\section{Las mejores prácticas de los profesionales}

Cumplir con las responsabilidades profesionales es una de las principales, si no la principal, obligación en toda profesión. Para satisfacer esta exigencia, es necesario tener una buena formación teórica y práctica, mantenerse al día, estar a la altura de los estándares profesionales y actuar de acuerdo con el código deontológico de la profesión. Aun así, estos componentes de la responsabilidad profesional, con ser necesarios, no son suficientes, porque los profesionales que trabajan en el campo de la discapacidad intelectual y de la discapacidad del desarrollo estrechamente relacionada (DID) deben además hacer gala de otras tres mejores prácticas profesionales: mostrar respeto por la persona, actuar con prácticas basadas en la evidencia y realizar la evaluación del efecto conseguido.

\section{Respeto por la persona}

Las mejores prácticas profesionales empiezan con una actitud basada en el respeto profundo por la persona. El respeto se caracteriza por centrar la atención en la persona, mostrar interés por ella, respetar los derechos humanos y legales que la asisten y realizar prácticas centradas en la persona que sirvan para mejorar su bienestar y autonomía personal. Esta forma de pensar y actuar está recogida en la Convención sobre los Derechos de las Personas con Discapacidad de las Naciones Unidas (2006). 
Tal como se establece en el artículo 1 de esta convención, "El propósito [de la Convención] es promover, proteger y garantizar el disfrute completo e igual de los derechos humanos y de las libertades por todas las personas con discapacidad, así como promover el respeto a su dignidad inherente".

En tanto que fundamento para las mejores prácticas de los profesionales, el respeto por la persona conlleva las siguientes acciones (Schalock y Luckasson, 2014): a) apoyar la autonomía personal, b) informar a la persona sobre los asuntos relevantes para su vida, incluidos el diagnóstico, la protección legal de la que goza y los derechos que le corresponden, c) hacer partícipe a la persona en el desarrollo y prestación de los servicios y apoyos que recibe, $d$ ) darle oportunidades para conseguir un mayor desarrollo personal, relaciones interpersonales, inclusión social y participación en el entorno de referencia, e) atender al bienestar emocional de la persona y a la necesidad de sentirse segura y protegida, f) atender a su bienestar físico, incluida la salud, la seguridad y la integridad física y g) explicarle la importancia que tiene la información y la forma en la que esta puede ayudarle a tomar decisiones que estén de acuerdo con sus valores y creencias.

\section{Estándares profesionales}

En tanto que componente de las mejores prácticas, en todas las profesiones existen estándares profesionales que constituyen el fundamento de los currículos para la formación teórico-práctica, de la evaluación de la práctica diaria de los miembros de la profesión y de la preparación personal como profesional. Son estándares importantes para estos profesionales los siguientes: a) utilizar estrategias para la obtención de información correcta y actualizada, b) obtener el consentimiento, incluidos los tres elementos principales del mismo: capacidad, información y voluntariedad, c) utilizar los datos de carácter personal de forma adecuada, d) evitar los conflictos de intereses y f) ser consciente del nivel de competencia en determinadas áreas y actuar solo en aquellas áreas en las que se tiene suficiente competencia (Schalock y Luckasson, 2014).

\section{Ética profesional}

La mayoría de las directrices éticas pueden resumirse enunciando las siguientes obligaciones: ser competente, ser profesional y mostrar responsabilidad científica, mostrar respeto por los derechos y la dignidad de la persona, interesarse por el bienestar de los demás y colaborar en el entorno inmediato y, en general, en la sociedad. Estas cinco obligaciones nos sirven para describir un sistema de comportamiento moral y el conjunto de normas de conducta que se reconocen y aceptan con respecto a un determinado colectivo de personas. Tal sistema de comportamiento y de normas morales se refleja en tres principios éticos: a) justicia (dar a todos el mismo trato), b) beneficencia (hacer el bien) y c) autonomía (respetar el derecho que asiste a toda persona de controlar las acciones que le afectan de forma directa). 


\section{Prácticas basadas en la evidencia}

Las prácticas basadas en la evidencia son acciones que se basan en la mejor evidencia disponible y se utilizan como base para la toma de decisiones clínicas, de gestión y organización y con el desarrollo y puesta en práctica de las diferentes políticas. La mejor evidencia actual es la información que se obtiene de fuentes fidedignas mediante métodos válidos y fiables. Esta información se basa en teorías o razonamientos claramente articulados y con suficiente apoyo empírico (Claes et al., 2014; Schalock y Verdugo, 2012; Schalock et al., 2011). Recientemente, el concepto de prácticas basadas en la evidencia se ha ampliado desde el punto de vista tradicional centrado en las modalidades de intervención y tratamiento a otras áreas, tales como los resultados obtenidos por la organización (van Loon et al., 2013), las estrategias individuales de apoyo (Claes et al., 2014; Horner et al., 2005) y el juicio clínico. En el caso de este último, las prácticas basadas en la evidencia se basan en la formación teórico-práctica y en la experiencia del clínico, en el conocimiento específico de la persona y de su entorno, en conjuntos extensos de datos y en el uso de las habilidades para el pensamiento crítico (Schalock y Luckasson, 2014).

\section{Evaluación del efecto}

Los profesionales tienen la obligación, como mejor práctica, de evaluar el efecto conseguido con los diferentes servicios y apoyos que proporcionan a las personas. La evaluación del efecto se centra en los resultados obtenidos con los servicios y apoyos, implica la valoración de las conductas relevantes para la persona y/o su familia y se basa en la multidimensionalidad de las personas y las familias.

La metodología que se utiliza en la evaluación del efecto incluye los estudios aleatorizados y controlados, múltiples estudios sobre los valores iniciales, diseños de un solo sujeto, enfoques cualitativos, encuestas sobre consumo y utilización y estudios de casos (Claes et al., 2014). Estos métodos se utilizan para valorar el efecto de los servicios y apoyos que se prestan en:

- Las áreas de las actividades vitales, tales como la vida en casa, la vida en el entorno inmediato situado fuera de casa, el aprendizaje y la formación a lo largo de toda la vida, el empleo, la salud y la seguridad, las actividades sociales y la protección y la defensa legal de la persona (Thompson et al., 2014).

- Las dimensiones del funcionamiento humano, incluido el funcionamiento intelectual, la conducta adaptativa, la salud, la participación y el contexto (Luckasson y Schalock, 2013).

- Los indicadores de cambio físico o conductual relacionados con situaciones médicas o conductuales excepcionales (Schalock et al., 2011).

- La calidad de las puntuaciones en los diferentes dominios vitales relacionados con el desarrollo personal, la autodeterminación, la inclusión social, las relaciones 
interpersonales, los derechos, el bienestar emocional, el bienestar físico y el bienestar material (Schalock y Verdugo, 2012).

- La calidad familiar de las puntuaciones de los diferentes dominios vitales (p. ej., las interacciones familiares, la crianza, el bienestar emocional, el desarrollo personal, el bienestar físico, el bienestar material, la implicación en el entorno inmediato fuera de casa y los apoyos relacionados con discapacidad) (Summers et al., 2005).

Los resultados de la evaluación del efecto se pueden utilizar para diferentes propósitos, los principales de los cuales son: la comunicación con los participantes clave y las personas afectadas o interesadas, la notificación de puntos de parámetros a efectos comparativos, reunir evidencia para las prácticas basadas en la evidencia, generar conocimiento y mejorar las competencias de los profesionales.

\section{Mejores prácticas de las organizaciones}

Las mejores prácticas de las organizaciones empiezan con el compromiso de ser una organización basada en valores que sea, a la vez, eficaz y eficiente en la prestación de los servicios y apoyos a los usuarios. La efectividad se define como el grado en el cual la organización consigue los resultados que se ha propuesto, mientras que la eficiencia se define como la capacidad de conseguir los resultados planificados por la organización en relación con el gasto de los recursos disponibles (Schalock y Verdugo, 2012).

\section{Valores}

Los valores en los que se basan las mejores prácticas de la organización están relacionados tanto con los servicios y apoyos que recibe el usuario como con los profesionales que prestan tales servicios y apoyos. Estos valores incluyen la equidad en la prestación de servicios y apoyos, la implicación y participación de los usuarios, la inclusión, la autodeterminación, la responsabilidad de actuar según lo que se es y los derechos legales. Algunos de estos valores están fuertemente anclados en la vida en general (Schalock y Keith, en prensa); algunos reflejan principios centrales de la discapacidad (Turnbull y Stowe, 2001; Shogren et al., 2014); y, por último, otros son propios de la Convención sobre los Derechos de las Personas con Discapacidad (Naciones Unidas, 2006).

Aunque lo que se ha indicado en los párrafos anteriores puede sugerir que estos valores tienen que ver solo con las personas con discapacidad, esto no es así. Los valores en los que se basan las mejores prácticas son genéricos en el sentido de que son canalizados y filtrados a través de equipos de alto rendimiento, que están indisolublemente ligados a las mejores prácticas de las organizaciones de las que trata este apartado: equipos de alto rendimiento, paradigma de los apoyos, evaluación de los 
resultados obtenidos y mejora continua de la calidad. Además, la motivación laboral y la satisfacción con el trabajo entre los profesionales se ven directamente influidas por el grado en el cual tales profesionales sienten que estos valores son aplicables a sí mismos y a su situación laboral (Hastings, 2010; Hewitt y Larson, 2007; Lunsky et al., 2014; Reinders y Schalock, 2014).

\section{Equipos de alto rendimiento}

Una característica de la época de las transformaciones es que las organizaciones se están haciendo más dinámicas, racionalizadas y funcionales debido al cambio que se está produciendo desde una estructura organizativa vertical a una estructura horizontal. Este aumento de la funcionalidad y de la racionalidad se acompaña de un uso cada vez mayor de los enfoques y métodos colaborativos en el liderazgo y gestión de la organización, en las prácticas de prestación de servicios y en la formación y puesta en práctica de equipos de alto rendimiento (Schalock y Verdugo, 2012, 2013). Los equipos de alto rendimiento son: a) grupos de trabajo estructurados horizontalmente que se basan en el trabajo en equipo, en la sinergia, en la elevación del listón del rendimiento, en la responsabilidad de actuar en función que quiénes son y cómo son, y en la promoción de la cultura del aprendizaje y del conocimiento compartido; b) grupos que se caracterizan por su implicación, compromiso, información, organización, responsabilidad y capacidad de tomar decisiones (autonomía); y c) grupos actualmente organizados en torno a la utilización de las tres mejores prácticas: planificación de los apoyos y prestación de los mismos a nivel individual, desarrollo de los recursos humanos a nivel de los profesionales de la organización y mejora de la calidad a nivel de la organización. Estas mejores prácticas se observan en los equipos de apoyo, en los equipos de recursos humanos y en los equipos de mejora de la calidad.

- Los equipos de apoyo están formados por los receptores de los servicios, los familiares o tutor legal de la persona, los profesionales encargados de los apoyos directos, el coordinador de los apoyos y otros profesionales relevantes. Sus principales funciones y responsabilidades consisten en desarrollar, poner en práctica, supervisar y evaluar los Planes Individuales de Apoyo. Funcionan mediante un modelo socioecológico del funcionamiento humano, y utilizan estrategias de apoyo que hacen hincapié en la mejora del potencial humano, en el crecimiento personal, en la inclusión social, en la autonomía, en la equidad, en la autodeterminación y en la inclusión en el entorno inmediato fuera de casa.

- Los equipos de recursos bumanos están compuestos por personas formadas en recursos humanos, desarrollo de equipos o desarrollo personal o con amplia experiencia práctica en estos campos. Sus funciones y responsabilidades principales consisten en desarrollar, poner en práctica, supervisar y evaluar el Plan de Desarrollo del Equipo. Trabajan en un contexto de desarrollo humano y de dinámica de grupos y utilizan estrategias de desarrollo de equipos en las que se hace hincapié en la mejora de la profesionalidad, del liderazgo, de la estabilidad

(C) Ediciones Universidad de Salamanca

Siglo Cero, vol. 46 (1), n. ${ }^{\circ} 253,2015$, enero-marzo, pp. 7-23 
emocional, de las habilidades para las relaciones interpersonales, de la inventiva para la resolución de problemas y del desarrollo de los recursos personales.

- Los equipos para la mejora de la calidad están formados por personas que tienen amplios conocimientos sobre las políticas, las prácticas y los sistemas de información de la organización y están implicadas en la ejecución de las prácticas de esta. Sus principales funciones y responsabilidades consisten en desarrollar, poner en práctica, supervisar y evaluar el Plan de Mejora de la Calidad. Trabajan dentro de un marco de mejora continua de la calidad y ponen el acento en un enfoque de control equilibrado de la gestión y la mejora de la calidad que consiste en cuatro perspectivas del rendimiento: la del usuario y la del crecimiento, la de los análisis económicos y la de los procesos internos de la organización.

\section{Paradigma de los apoyos}

Desde mediados de la década de 1980, el paradigma de los apoyos ha servido para conectar las políticas relacionadas de planificación centrada en la persona basada en los puntos fuertes y valía de esta con las necesidades de apoyo evaluadas, el desarrollo personal y las oportunidades de crecimiento, la inclusión en el entorno inmediato fuera de casa, la autodeterminación y la autonomía. Como mejor práctica, el paradigma de los apoyos proporciona un punto de vista compartido que conecta las prácticas en el nivel individual con las prioridades y la misión de la organización en el nivel del entorno social y con las políticas y toma de decisiones en el nivel de los sistemas (Thompson et al., 2014).

En el nivel individual, el paradigma de los apoyos proporciona a los equipos de apoyo una forma de pensar/modelo mental que enfatiza: a) los puntos fuertes y valía de la persona, b) las metas de la persona y las necesidades de apoyo evaluadas en lo que respecta a las principales áreas de actividad vital y a las situaciones médicas y conductuales excepcionales, c) las características personales y los factores del entorno que afectan al funcionamiento de los seres humanos y el papel que desempeñan los apoyos individualizados en la reducción de la discrepancia entre las demandas del entorno y las competencias personales, d) un sistema de apoyos que incluye los basados en lo cognitivo y en la tecnología, estrategias para la adquisición de competencias, acomodación al entorno, protésica, apoyos conductuales, apoyos naturales y prácticas profesionales y e) la provisión de estrategias específicas de apoyos y la evaluación de su efecto sobre los resultados personales evaluados.

En el nivel de la organización, el paradigma de los apoyos proporciona la base para las cuatro mejores prácticas. En primer lugar, ofrece el marco para que la organización lleve a cabo una evaluación estandarizada de las necesidades de apoyo de la persona en todas las áreas de actividad vital. La evaluación da lugar a un perfil del patrón e intensidad de los apoyos que se necesitan que, cuando se suman, se puede utilizar para diferentes propósitos, entre otros, la elaboración de informes, la toma de decisiones presupuestarias, la formación de los profesionales y la elaboración de parámetros a

(C) Ediciones Universidad de Salamanca

Siglo Cero, vol. 46 (1), n. ${ }^{\circ} 253,2015$, enero-marzo, pp. 7-23 
efectos de comparación. En segundo lugar, el paradigma de los apoyos permite a la organización desarrollar un procedimiento organizado para la prestación de apoyos que: a) organiza las posibles estrategias de apoyos en un sistema a través del cual se pueden planificar y llevar a cabo los apoyos personalizados sobre la base de las necesidades de apoyos individualmente evaluadas, b) ofrece un marco que permite alinear la obtención y aplicación de los apoyos personalizados a partir de las fuentes de apoyo y c) proporciona el marco para evaluar el efecto de los apoyos personalizados sobre el funcionamiento de la persona y los resultados personales.

En tercer lugar, el paradigma de los apoyos proporciona los parámetros principales para los sistemas de información de la organización. Concretamente, ofrece información relacionada con los apoyos obtenida a partir del mismo instrumento para la toma de decisiones basada en datos; contribuye a facilitar la racionalización del proceso de planificación de los apoyos; identifica los recursos de los que se dispone para las necesidades de apoyos evaluadas, y permite informar, supervisar, generar parámetros comparativos, evaluar y mejorar la calidad de forma continua. Por último, cuando se combina con la evaluación de los resultados obtenidos, el paradigma de los apoyos proporciona a las organizaciones un modelo conceptual y cuantitativo que sirve para alinear mejor el sistema de prestación de servicios. En la Tabla I se presentan los indicadores más importantes de este alineamiento.

\begin{tabular}{|l|l|l|l|}
\hline \multicolumn{2}{|c|}{ TABLA 1. Principales indicadores que representan un sistema de prestación de servicios alineado } \\
\hline $\begin{array}{c}\text { Nivel de los sistemas de } \\
\text { prestación de servicios }\end{array}$ & \multicolumn{1}{|c|}{$\begin{array}{c}\text { Componentes de } \\
\text { entrada }\end{array}$} & $\begin{array}{c}\text { Componentes de } \\
\text { procesamiento }\end{array}$ & Componentes de salida \\
\hline Nivel individual & $\begin{array}{l}\text { Metas personales y } \\
\text { necesidades de apoyos } \\
\text { evaluadas }\end{array}$ & Sistema de apoyos & Resultados personales \\
\hline Nivel de la organización & $\begin{array}{l}\text { Políticas basadas en } \\
\text { valores } \\
\text { Recursos }\end{array}$ & $\begin{array}{l}\text { Servicios de la } \\
\text { organización } \\
\text { Estrategias de gestión }\end{array}$ & $\begin{array}{l}\text { Resultados personales } \\
\text { Resultados de la } \\
\text { organización }\end{array}$ \\
\hline Nivel del sistema & $\begin{array}{l}\text { Políticas basadas en } \\
\text { valores } \\
\text { Recursos }\end{array}$ & $\begin{array}{l}\text { Marco para la prestación } \\
\text { de los servicios }\end{array}$ & $\begin{array}{l}\text { Total de los resultados } \\
\text { de la organización } \\
\text { Indicadores de cambio } \\
\text { en el sistema }\end{array}$ \\
\hline
\end{tabular}

\section{Evaluación de los resultados obtenidos}

Uno de los retos más importantes de las organizaciones de DID es demostrar que son eficaces tanto en lo que se refiere a la consecución de los resultados como a la eficiencia en lo referente a la consecución de tales resultados en relación con la utilización de los recursos disponibles. Superar este reto, que exige a las organizaciones 
evaluar y notificar los resultados obtenidos, es difícil debido a una serie de razones. En primer lugar, los servicios humanos tradicionalmente se han centrado en el proceso (es decir, en lo que se hace) y no en los resultados conseguidos (es decir, en el efecto que tiene lo que se hace). En segundo lugar, la evaluación de los resultados obtenidos requiere un modelo conceptual y de medición claro que incorpore los valores, las mejores prácticas y los sistemas de información. En tercer lugar, tradicionalmente la evaluación ha sido una actividad externa relacionada con la financiación, la acreditación y homologación, los permisos y la certificación, en vez de un proceso interno y colaborativo centrado en la capacidad de generar calidad y de mejorarla de forma continua. La superación de este problema se ha visto facilitada por dos cambios importantes que se han producido recientemente. En primer lugar, se han desarrollado modelos conceptuales y de medición en el nivel tanto individual como de la organización que proporcionan un marco claro para identificar las diferentes categorías de los resultados cuantificables (Schalock y Verdugo, 2012). En segundo lugar, las organizaciones de servicios humanos están empezando a incorporar un enfoque participativo y colaborativo para la evaluación y el cambio en la organización (Schalock et al., 2014).

Desde la perspectiva de las mejores prácticas, es necesario considerar la evaluación de los resultados obtenidos en el marco de un modelo lógico de programa formado por tres componentes: entradas, procesamiento y salidas/resultados. En este marco, las entradas son los valores y los recursos, tales como los conocimientos explícitos e implícitos, el tiempo, el capital social y económico y la tecnología. El procesamiento consiste en los apoyos específicos, la mejora de la calidad y las estrategias para el desarrollo de los equipos que se utilizan para mejorar el rendimiento de los profesionales, de los equipos y de la organización; reforzar la eficacia y la eficiencia del marco de prestación de servicios, y utilizar estrategias de gestión estructuradas horizontalmente. Las salidas son las medidas que sirven para evaluar la eficacia y la eficiencia de la organización en términos de las cuatro perspectivas basadas en el rendimiento: la del usuario y las de la organización (crecimiento, análisis económicos y procesos internos). En la Tabla 2 se presentan ejemplos de resultados (salidas) de una organización.

\begin{tabular}{|c|l|l|}
\hline \multicolumn{2}{|c|}{ TABLA 2. Diferentes perspectivas sobre los resultados de la organización } \\
\hline $\begin{array}{c}\text { Resultados } \\
\text { de la organización }\end{array}$ & \multicolumn{1}{|c|}{$\begin{array}{c}\text { Perspectiva basada } \\
\text { en el rendimiento }\end{array}$} & \multicolumn{1}{c|}{$\begin{array}{c}\text { Ejemplos de resultados } \\
\text { de la organización }\end{array}$} \\
\hline Eficacia & Usuario & $\begin{array}{l}\text { - Mejora de los resultados personales } \\
- \text { Sistemas de apoyos puestos en marcha y en funciona- } \\
\text { miento }\end{array}$ \\
& Crecimiento & $\begin{array}{l}\text { Incremento de los opciones de programas } \\
- \text { Aumento de la implicación de los profesionales en los } \\
\text { equipos de alto rendimiento }\end{array}$ \\
\hline
\end{tabular}

(C) Ediciones Universidad de Salamanca

Siglo Cero, vol. 46 (1), n. ${ }^{\circ} 253,2015$, enero-marzo, pp. 7-23 


\begin{tabular}{|l|l|l|}
\hline $\begin{array}{c}\text { Resultados } \\
\text { de la organización }\end{array}$ & \multicolumn{1}{c|}{$\begin{array}{c}\text { Perspectiva basada } \\
\text { en el rendimiento }\end{array}$} & \multicolumn{1}{c|}{$\begin{array}{c}\text { Ejemplos de resultados } \\
\text { de la organización }\end{array}$} \\
\hline Eficiencia & Análisis económicos & $\begin{array}{l}\text { - Reducción de los gastos generales (indirectos) } \\
- \text { Reducción del coste económico por unidad de servi- } \\
\text { cio/apoyo }\end{array}$ \\
& Procesos internos & $\begin{array}{l}\text { Modelos lógicos de programa que se utilizan para ali- } \\
\text { near los procesos y las funciones } \\
\end{array}$ \\
& $\begin{array}{l}\text { Sistemas de información basados en páginas web en } \\
\text { funcionamiento para generar información basada en el } \\
\text { rendimiento }\end{array}$ \\
\hline
\end{tabular}

Los resultados de la evaluación de las salidas generalmente se utilizan para elaborar informes, generar parámetros comparativos, supervisar, evaluar y mejorar la calidad de forma continua. Además, en muchas organizaciones transformadas proporcionan información para poner en práctica un método de anotaciones equilibrado que sirve para planificar y evaluar los programas y constituyen la base de los procesos de resolución de problemas sin afectar a los valores y metas de la organización.

\section{Mejora continua de la calidad}

La mejora continua de la calidad (MCC) es un proceso interno, colaborativo y transformativo que incluye la valoración, la planificación, la puesta en práctica y la evaluación. La MCC conlleva procesos paralelos en el nivel individual, de los equipos y de la organización. Para las personas, el punto principal es la mejora de los resultados personales; para los equipos de alto rendimiento lo importante es la mejora del rendimiento del equipo; y para la organización, la mejora de la eficacia y de la eficiencia.

En tanto que una de las mejores prácticas, la MCC es más efectiva cuando se basa en un bucle de mejora continua de la calidad compuesto por la valoración, la planificación, la puesta en práctica y la evaluación. Este concepto se explica en la Figura 2 y se operativiza en la Tabla 3.

El componente 'planificación' de la MCC requiere que
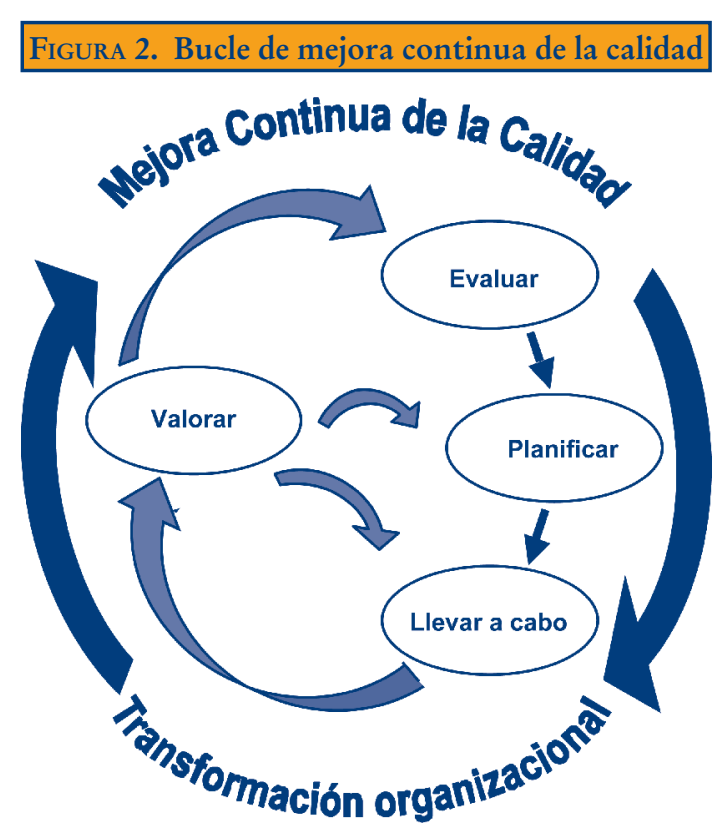

(C) Ediciones Universidad de Salamanca

Siglo Cero, vol. 46 (1), n. 253,2015 , enero-marzo, pp. 7-23 
cada equipo utilice un formato de planificación centrado en los resultados obtenidos para sintetizar los resultados de la valoración y desarrollar los componentes de los apoyos individuales, y la mejora de la calidad y el plan de desarrollo del equipo. Este proceso se resume en el formato de planificación prototípico que se presenta en la Tabla 4. Debe tenerse en cuenta que en la Tabla 4 NO se presenta un formato para la planificación que sea obligatorio para todas las organizaciones. Se trata más bien de una estructura cuya función es la de orientar al equipo para planificar, poner en práctica, supervisar y evaluar los planes en el contexto de un marco centrado en los resultados obtenidos. Las seis columnas muestran los pasos lógicos y secuenciales implicados.

\begin{tabular}{|c|c|c|c|}
\hline \multicolumn{4}{|c|}{ TABLA 3. Componentes de la MCC relacionados con las personas, los equipos y la organización } \\
\hline $\begin{array}{c}\text { Componente del bucle } \\
\text { de mejora continua } \\
\text { de la calidad }\end{array}$ & $\begin{array}{c}\text { Nivel } \\
\text { de las personas }\end{array}$ & $\begin{array}{c}\text { Nivel } \\
\text { de los equipos }\end{array}$ & $\begin{array}{c}\text { Nivel } \\
\text { de la organización }\end{array}$ \\
\hline Valoración & $\begin{array}{l}\text { Metas personales y } \\
\text { necesidad de apoyos } \\
\text { evaluadas (SIS)* }\end{array}$ & $\begin{array}{l}\text { Metas del equipo y } \\
\text { necesidades evaluadas } \\
\text { de desarrollo del equipo } \\
(\mathrm{CFI})^{* *}\end{array}$ & $\begin{array}{l}\text { Metas de la organización } \\
\text { y necesidades evaluadas } \\
\text { de mejora de la calidad } \\
(\mathrm{OEES})^{* * *}\end{array}$ \\
\hline Planificación & $\begin{array}{l}\text { Plan Individual de Apoyos } \\
\text { (Equipo de Apoyos) }\end{array}$ & \begin{tabular}{|l|} 
Plan de Desarrollo del \\
Equipo (Equipo de Recursos \\
Humanos)
\end{tabular} & $\begin{array}{l}\text { Plan de Mejora de la } \\
\text { Calidad (Plan de Mejora } \\
\text { de la Calidad) }\end{array}$ \\
\hline Puesta en práctica & $\begin{array}{l}\text { Puesta en práctica y } \\
\text { supervisión (Equipo de } \\
\text { Apoyo) }\end{array}$ & $\begin{array}{l}\text { Puesta en práctica y } \\
\text { supervisión (Equipo de } \\
\text { Recursos Humanos) }\end{array}$ & $\begin{array}{l}\text { Puesta en práctica y } \\
\text { supervisión (Equipo de } \\
\text { Mejora de la Calidad) }\end{array}$ \\
\hline Evaluación & $\begin{aligned} \text { - } & \text { Estado de los objetivos } \\
& \text { con respecto a los apoyos } \\
\text { - } & \text { Resultados personales } \\
& \text { (GENCAT; INICO } \\
& \text { FEAPS; San Martín)**** }\end{aligned}$ & 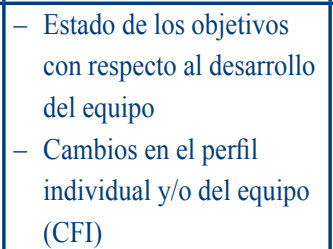 & \begin{tabular}{|l} 
- Estado de los \\
objetivos con respecto \\
a la mejora de la \\
calidad \\
- Índices de cambio \\
OEES (OEES)
\end{tabular} \\
\hline \multicolumn{4}{|c|}{$\begin{array}{l}\text { *Supports Intensity Scale [Escala de Intensidad de los Apoyos] } \\
* \text { Championship Formula Inventory [Inventario de la Fórmula del Campeón] } \\
* \because \% \text { Organization Effectiveness and Efficiency Scale [Escala de Eficacia y Eficiencia de la Organización] } \\
* \ldots \% \text { GENCAT o INICO FEAPS; San Martín }\end{array}$} \\
\hline
\end{tabular}

(C) Ediciones Universidad de Salamanca

Siglo Cero, vol. 46 (1), n. ${ }^{\circ} 253,2015$, enero-marzo, pp. 7-23 


\begin{tabular}{|c|c|c|c|c|c|}
\hline \multicolumn{6}{|c|}{ TABLA 4. Formato de planificación prototípico } \\
\hline (1) & (2) & (3) & (4) & (5) & (6) \\
\hline $\begin{array}{l}\text { Resultados } \\
\text { obtenidos }\end{array}$ & \begin{tabular}{|l|} 
Metas y \\
necesidad \\
evaluadas más \\
importantes
\end{tabular} & Estrategias & Objetivos & $\begin{array}{l}\text { Requerimientos } \\
\text { para la puesta } \\
\text { en práctica }\end{array}$ & $\begin{array}{l}\text { Requerimientos } \\
\text { para la } \\
\text { supervisión y la } \\
\text { evaluación }\end{array}$ \\
\hline 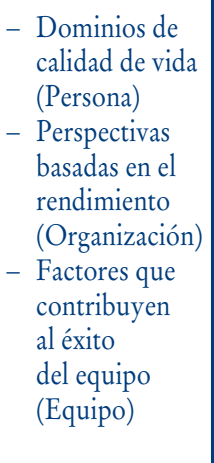 & 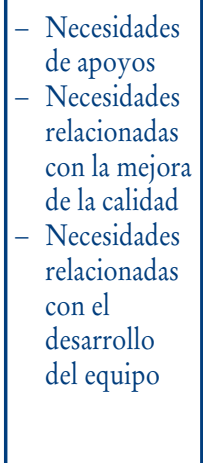 & \begin{tabular}{|l} 
- Estrategias \\
individuales \\
de apoyos \\
- Estrategias de \\
mejora de la \\
calidad \\
- Estrategias de \\
desarrollo del \\
equipo
\end{tabular} & $\begin{array}{l}\text { - Verbo activo } \\
\text { (la base para } \\
\text { poner en } \\
\text { práctica) } \\
\text { - Estrategia } \\
\text { específica } \\
\text { (la base para } \\
\text { el cambio y la } \\
\text { supervisión) } \\
\text { - Resultado } \\
\text { previsto (la } \\
\text { base para la } \\
\text { evaluación) }\end{array}$ & $\begin{array}{l}\text { - Quién } \\
\text { - Qué } \\
\text { - Cuándo } \\
\text { - Cómo }\end{array}$ & $\begin{array}{l}\text { - Quién } \\
\text { - Qué } \\
\text { - Cuándo }\end{array}$ \\
\hline
\end{tabular}

En tanto que una de las mejores prácticas, la MCC debe abordarse desde la perspectiva de que la calidad es un asunto que afecta a todas las áreas de la organización. Por lo tanto, es necesario utilizar un enfoque holístico y exhaustivo, no solo en lo que se refiere a los productos, servicios o programas, sino también en lo referente a los equipos y a los procesos y procedimientos que la organización incorpora en sus actividades. Asimismo, la MCC debe ser parte de la cultura profunda de la organización a fin de que sea una realidad. El enfoque basado en las mejores prácticas para la MCC que acabamos de describir requiere un procedimiento de puesta en práctica que sea sensible a la receptividad de la organización, sea percibido como un elemento capaz de hacer avanzar la competitividad y la posición de la organización y, a la vez, de proporcionar un conjunto de valores a las personas implicadas en la organización o afectadas por sus actividades. Debe, además, poder ser comprendido fácilmente y enseñado al personal por los equipos de consultoría y formación, pero, a la vez, debe ser realista y tener en cuenta las limitaciones de recursos de la organización.

Papel de los profesionales que intervienen directamente en la prestación de los apoyos en el desarrollo y ejecución de las mejores prácticas de las organizaciones y de los profesionales

La relación recíproca entre las mejores prácticas de las organizaciones y de los profesionales proporciona la base para intentar resolver uno de los retos más complicados a los que se enfrentan actualmente las organizaciones de DID: el desarrollo de 
los recursos humanos y la utilización de las mejores prácticas por parte de los profesionales de las organizaciones. Resolver este problema requiere en parte crear dentro de la organización un entorno cultural que sirva no solo para mejorar los servicios y los apoyos que la organización proporciona a los usuarios, sino también para ofrecer oportunidades de crecimiento a los profesionales que están directamente implicados en la prestación de los apoyos, de tal forma que estén más comprometidos con su trabajo, sean más eficaces y eficientes y se sientan más respetados como miembros integrantes de un equipo profesional.

La necesidad de aumentar la implicación de los profesionales que participan directamente en la prestación de los apoyos en la ejecución de las mejores prácticas está relacionada no solo con el esquema mental según el cual "los profesionales son los que más saben y son los principales actores en el proceso de toma de decisiones”, sino también con la transformación en la cultura de los servicios profesionales que se prestan a las personas con DID. Tradicionalmente, los profesionales expertos (p. ej., médicos, psicólogos, personal de enfermería, educadores y trabajadores sociales) eran las personas que tenían más autoridad en las organizaciones. Durante muchos años, estos expertos han proporcionado el conocimiento, los estándares, los protocolos y las prácticas que servían de base a los procesos de atención y tratamiento con un grado razonable de autonomía e independencia profesional. No obstante, a partir de la última década del siglo XX, esta situación empezó a cambiar, y ahora estos profesionales son percibidos más como empleados de una organización o agencia que actúan en interés de las metas y objetivos de la organización a la que pertenecen o con la que colaboran y, además, este cambio, ha afectado también a la percepción de las personas con discapacidad, que ahora son percibidas como usuarios o clientes (Noordegraaf, 2007; Reinders, 2008). Al mismo tiempo, hemos asistido a la aparición de una nueva concepción de la gestión de lo público en la que el mercado se considera el principal instrumento regulador en la esfera de lo público. Los bienes públicos, tales como el bienestar de las personas con DID, se perciben ahora como productos sometidos a los mecanismos característicos del mercado: oferta, demanda y precio. Los mecanismos de la nueva gestión de lo público son actualmente los mecanismos propios del mercado, la descentralización, el control de la calidad, la medición del rendimiento, el énfasis en los resultados, la eficiencia y la relación coste-efectividad. Como consecuencia, las personas profesionalmente formadas tiene que dedicar una cantidad considerable de tiempo en los detalles referidos a la administración y la documentación (DiRita et al., 2008). El resultado final es que disponen de menos tiempo para la prestación de servicios directos.

Con esta transformación en la función y en la disponibilidad de tiempo para la prestación de los servicios directos de los profesionales tradicionales, el papel que los profesionales implicados en la prestación directa de los apoyos desempeñan en las organizaciones está experimentando también un cambio. Como miembros de un equipo de alto rendimiento, ponen en práctica estrategias de apoyo personalizadas, participan en la evaluación de los resultados obtenidos y colaboran en las estrategias de mejora de la calidad (Hastings, 2007; Hewitt y Larson, 2007; Lunsky et al., 2014; Reinders y Schalock, 2014; Schalock y Verdugo, 2012). 
¿Bajo qué condiciones, por tanto, puede 'un profesional directamente implicado en la prestación de los apoyos' ser considerado 'un profesional directamente implicado en la prestación de los apoyos'? Si bien se trata de un asunto de competencias profesionales y, por tanto, legal, que en muchos casos tiene que ver los títulos universitarios y con la acreditación, exponemos a continuación una serie de ideas y directrices basadas en el trabajo de Schalock y Luckasson (2014) sobre el juicio clínico y el papel del clínico en la DI. El grado de profesionalismo que se otorga a un trabajador directamente implicado en la prestación de los apoyos depende de: a) la complejidad del asunto y de la cantidad y tipo de información de la que se dispone; b) la cualificación, formación teórico-práctica, experiencia y nivel de experto de la persona y del grado en el cual la cualificación se adapta a la(s) mejor(es) práctica(s) en cuestión; c) las funciones y responsabilidades específicas de la persona (p. ej., algunas funciones, tales como el diagnóstico y/o la prescripción de medicamentos u otros tratamientos, están reguladas por ley); y d) que se espera en la organización del profesional en términos de estándares prácticos.

Con independencia del grado de profesionalismo aportado por los trabajadores directamente implicados en la prestación de los apoyos, el hecho es que los receptores de los apoyos pasan aproximadamente el $80 \%$ del tiempo en el que están despiertos en compañía de estos trabajadores. En consecuencia, su implicación es esencial para que la organización consiga poner en práctica los componentes de las mejores prácticas que se presentan en la Figura 1 y que hemos explicado previamente. Reforzar el profesionalismo de los trabajadores directamente implicados en la prestación de los apoyos se puede conseguir mediante la contratación de servicios, los programas de formación continua y los programas de desarrollo de los recursos humanos centrados en el desarrollo de los equipos y en el desarrollo personal. Este refuerzo es, además, una de las funciones clave que los líderes de la transformación pueden desempeñar como tutores, dirigentes, coachers y formadores, inspirando, otorgando mayor autonomía y capacidad de decisión, colaborando y asesorando.

La naturaleza recíproca de las mejores prácticas de las organizaciones y de los profesionales

La naturaleza recíproca de las mejores prácticas de las organizaciones y de los profesionales tiene un efecto compartido en ambos niveles. ¿En qué consiste este efecto compartido? A partir de la descripción de las diez mejores prácticas que se hace en la Figura 1 y que hemos explicado, aparecen claramente cinco efectos positivos. El primer efecto es un enfoque de la relación con los usuarios y con los profesionales basado en el respeto y en los valores centrados en las personas y en la organización que sirven de guía a las políticas y a las prácticas de esta. En segundo lugar, las mejores prácticas proporcionan un mecanismo que contribuye a facilitar los cambios estructurales en la organización desde la estructura vertical basada en la autoridad a la estructura horizontal basada en la colaboración y la acción. En tercer lugar, el enfoque holístico de las mejores prácticas anima y motiva a los profesionales

(C) Ediciones Universidad de Salamanca

Siglo Cero, vol. 46 (1), n. ${ }^{\circ} 253,2015$, enero-marzo, pp. 7-23 
y a las organizaciones a basar la planificación y la puesta en práctica de los servicios y apoyos no solo en los estándares y la ética profesionales, sino también en las metas personales, en los apoyos personalizados y en las prácticas basadas en la evidencia. En cuarto lugar el énfasis puesto en las prácticas basadas en la evidencia, en la evaluación del efecto y en la evaluación de los resultados obtenidos hace que la organización se perciba a sí misma como generadora de conocimiento y dispuesta a valorar y compartir no solo lo que la organización hace, sino también los resultados conseguidos gracias a su esfuerzo. Por último, el énfasis en la evidencia, tal como se refleja en las prácticas basadas en la evidencia, en la evaluación del efecto y en la evaluación de los resultados obtenidos, añadido a la incorporación de la mejora continua de la calidad en la cultura de la organización, da lugar a que esta sea más eficaz, eficiente y sostenible gracias a un proceso de transformación interno y colaborativo.

En última instancia, las mejores prácticas de las organizaciones y de los profesionales no tienen que ver solo con la eficacia, la eficiencia y la sostenibilidad de la organización: las mejores prácticas tiene que ver también con la mejora de la vida de las personas, porque ofrecen la oportunidad de mejorar el funcionamiento y el bienestar personales. Con esta finalidad, las mejores prácticas de las organizaciones y de los profesionales son utilizadas por personas y organizaciones para el bien de la sociedad, para tratar con justicia a los individuos y su familia y para conseguir la integridad ética en nuestro campo de actuación.

\section{Referencias bibliográficas}

Claes, C., van Loon, J., Vandevelde, S. y Schalock, R. L. (2014). An integrative approach to evidence based practices. Evaluation and Program Planning (in press).

DeRita, B. A., Parmenter, T. R. y Stancliffe, R. J. (2008). Utility, economic rationalism, and the circumscription of agency. Journal of Intellectual Disability Research, 52, 618-624.

Hastings, R. P. (2010). Support staff working in intellectual disability services: The importance of relationships and positive experiences. Journal of Intellectual and Developmental Disability, 35, 207-210.

HewitT, A. y LARson, S. (2007). The direct workforce in community supports to individuals with developmental disabilities: Issues, implications, and promising practices. Mental Retardation and Developmental Disabilities, 13, 178-187.

Luckasson, R. y SCHAlock, R. L. (2013). Defining and applying a functionality approach to intellectual disability. Journal of Intellectual Disability Research, 57, 657-668.

Lunsky, Y., Hastings, R. P., Hensel, J., Arenovich, T. y Dewa, C. S. (2014). Perceptions of positive contributions and burnout in community developmental workers. Intellectual and Developmental Disabilities, 52, 249-257.

Mazzotti, V. L., Test, D. W. y Mustian, A. L. (2014). Secondary transition evidence-based practices and predictors: Implications for policy makers. Journal of Disability Policy Studies, 25 (1), 5-18.

NeUtens, J. J. y Rubinson, L. (2010). Research techniques for the health sciences. San Francisco, CA: Benjamin Cummins.

NoordegraAf, M. (2007). From 'pure' to 'hybrid' professionalism: Present-day professionalism in ambiguous public domains. Administration and Society, 39, 761-784. 
NoRwOOD, S. L. (2010). Research essentials: Foundations for evidence-based practices. Boston, MA: Pearson.

REINDERS, H. (2008). The transformation of human services. Journal of Intellectual Disability Research, 52, 564-571.

ReINDERs, H. S. y SCHAlock, R. L. (2014). How organizations can enhance the quality of life of their clients and assess their results: The concept of QOL enhancement. American Journal on Intellectual and Developmental Disabilities, 119, 291-302.

SCHALOCK, R. L. y KeITH, K. D. (in press). Cross-cultural quality of life: Enhancing the lives of persons with intellectual disability. Washington, DC: American Association on Intellectual and Developmental Disabilities.

Schalock, R. L. y Luckasson, R. (2014). Clinical judgment (2 ${ }^{\text {nd }}$ ed.). Washington, DC: American Association on Intellectual and Developmental Disabilities.

Schalock, R. L. y Verdugo, M. Á. (2012). A leadership guide for today's disabilities organizations: Overcoming challenges and making change happen. Baltimore, MD: Brookes Publishing Company.

Schalock, R. L. y Verdugo, M. Á. (2013). The transformation of disabilities organizations. Intellectual and Developmental Disabilities, 51, 273-286.

Schalock, R. L., Verdugo, M. Á. y Gómez, L. E. (2011). Evidence-based practices in the field of intellectual and developmental disabilities. Evaluation and Program Planning, 34, 273-282.

SHIRE, S. y KASARI, C. (2014). Train the trainer effectiveness trials of behavior intervention for individuals with autism: A systematic review. American Journal on Intellectual and Developmental Disabilities, 119, 436-451.

Shogren, K. A., LuCKasson, R. y SCHAlock, R. L. (2014). The definition of 'context' and its application in the field of intellectual disability. Journal of Policy and Practice in Intellectual Disabilities, 11, 109-116.

Summers, J. A., Poston, D. J., Turnbull, A. P., Marquis, J., Hoffman, L., Mannan, H. y WANG, M. (2005). Conceptualizing and measuring family quality of life. Journal of Intellectual Disability Research, 49 (10), 777-783.

Thompson, J. R., Schalock, R. L., Agosta, J., Teninty, L. y Fortune, J. (2014). How the supports paradigm is transforming the developmental disabilities service system. Inclusion, 2, 86-99.

Turnbull, H. R. y Stowe, M. J. (2001). A taxonomy for organizing the core concept according to their underlying principles. Journal of Disability Policy Studies, 112, 177-197.

United Nations (2006). Convention on the Rights of Persons with Disabilities. Retrieved from http://www.un.org/disabilities/convention

Van Loon, J. H. M., Bonham, G. S., Peterson, D. D., Schalock, R. L., Claes. C. y DecraMER, A. (2013). The use of evidence-based outcomes in systems and organization providing services and supports to persons with intellectual disability. Evaluation and Program Planning, 36, 80-87. 